\title{
Descriptions of Barriers to Self-Care by Persons with Comorbid Chronic Diseases
}

Elizabeth A. Bayliss, MD, MSPH ${ }^{1,4}$

Jobn F. Steiner, MD, MPH

Douglas H. Fernald, $M A^{4}$

Lori A. Crane, $\mathrm{PbD}, \mathrm{MPH}^{3}$

Deborab S. Main, $P b D^{1}$

${ }^{1}$ Department of Family Medicine, University of Colorado Health Sciences Center,

Denver, Colo

${ }^{2}$ Division of General Internal Medicine, University of Colorado Health Sciences Center, Denver, Colo

${ }^{3}$ Department of Preventive Medicine and Biometrics, University of Colorado Health Sciences Center, Denver, Colo

${ }^{4}$ Clinical Research Unit, Kaiser Permanente, Denver, Colo
Conflict of interest: None reported

\section{CORRESPONDING AUTHOR}

Elizabeth A. Bayliss, MD, MSPH

Kaiser Permanente Clinical Research Unit PO Box 378006

Denver, CO 80237-8006

elizabeth.bayliss@kp.org

\begin{abstract}
BACKGROUND Chronic medical conditions often occur in combination, as comorbidities, rather than as isolated conditions. Successful management of chronic conditions depends on adequate self-care. However, little is known about the self-care strategies of patients with comorbid chronic conditions.
\end{abstract}

OBJECTIVE Our objective was to identify perceived barriers to self-care among patients with comorbid chronic diseases.

METHODS We conducted semistructured personal interviews with 16 adults from 4 urban family practices in the CaReNet practice-based research network who self-reported the presence of 2 or more common chronic medical conditions. Using a free-listing technique, participants were asked, "Please list everything you can think of that affects your ability to care for your medical conditions." Responses were analyzed for potential barriers to self-care.

RESULTS Participants' responses revealed barriers to self-care, including physical limitations, lack of knowledge, financial constraints, logistics of obtaining care, a need for social and emotional support, aggravation of one condition by symptoms of or treatment of another, multiple problems with medications, and overwhelming effects of dominant individual conditions. Many of these barriers were directly related to having comorbidities.

CONCLUSIONS Persons with comorbid chronic diseases experience a wide range of barriers to self-care, including several that are specifically related to having multiple medical conditions. Self-management interventions may need to address interactions between chronic conditions as well as skills necessary to care for individual diseases.

Ann Fam Med 2003;1:15-21. DOI: 10.1370/afm.4

\section{BACKGROUND}

urrently, 125 million persons in the United States suffer from chronic medical conditions. ${ }^{1}$ The burden of chronic illness is magnified for many of them by the fact that chronic conditions often occur as comorbidities (coexisting medical conditions) rather than as single conditions. Of these 125 million, 60 million have multiple chronic conditions. ${ }^{1}$ These numbers are expected to increase to 157 million and 81 million, respectively, by the year 2020. ${ }^{1}$ The occurrence of comorbidity is age-dependent, with $69 \%$ of persons over age 65 having 2 or more chronic illnesses. ${ }^{2}$ It is estimated that more than $40 \%$ of people with a chronic condition have at least 1 comorbidity, ${ }^{2}$ and that persons aged 60 years and older have an average of 2.2 chronic conditions. ${ }^{3}$ Increasing numbers of chronic conditions have been associated with increased levels of disability and decreased levels of physical wellbeing. ${ }^{4,5}$ In addition, comorbidity has been associated with increased 
mortality, decreased quality of life and increased use of health services. ${ }^{6}$

Effective management of chronic illness is complex and requires significant participation by patients and their families. Day-to-day disease self-management has multiple components:

- Engaging in activities that promote physical and psychological health;

- Interacting with health care providers and adherence to treatment recommendations;

- Monitoring health status and making associated care decisions;

- Managing the impact of the illness on physical, psychological and social functioning. ${ }^{7,8}$

Any impediments to these processes may jeopardize successful disease management and thereby represent barriers to the self-care process.

Since levels of disability and functioning differ with the number of comorbid chronic diseases, and management of chronic disease depends largely on self-care, ${ }^{4,5,7}$ it is plausible that effective self-care of comorbid conditions differs from that required for single conditions. Persons with comorbid conditions may experience barriers to self-care that arise from the simultaneous demands of comorbidities. If so, self-management interventions geared towards single diseases may fail to address the priorities of persons with multiple medical conditions. Previous studies of patient perceptions of barriers to self-care have concentrated on single diseases. ${ }^{9-13} \mathrm{We}$ found no studies that assessed barriers to self-care specific to patients with more than 1 chronic disease.

Based on the hypothesis that patients with 2 or more chronic illnesses experience barriers to self-care that result from having comorbid chronic diseases, we designed a qualitative study to explore perceived barriers to self-care among persons with multiple chronic conditions. Because chronic conditions cover a broad spectrum of disease and disease severity, we chose to include in this exploratory study individuals who had 2 or more of a list of common chronic conditions. These diagnoses were chosen based on their prevalence and frequent reference in the literature on comorbidity. ${ }^{6}$

\section{METHODS}

\section{Study design}

We conducted a qualitative study using semistructured personal interviews.

\section{Study setting}

The study was conducted in 4 family medicine practices in metropolitan Denver, Colo, through the CaReNet primary care research network. CaReNet is a state-wide primary care research network of 23 primary care practices founded in 1997 with a commitment to serving disadvantaged populations. ${ }^{14}$

\section{Study subjects}

Sixteen subjects were recruited through advertisements placed in the practices. These advertisements, which were posted in waiting rooms and exam rooms of the practices, listed common chronic medical problems (osteoarthritis, coronary artery disease, chronic obstructive pulmonary disease, asthma, diabetes, hypertension, and congestive heart failure) and invited individuals who identified themselves as having 2 or more of these conditions to participate in a research interview. Potential participants were then screened by telephone for presence of any exclusion criteria (see below).

For the specific objectives of this study, we selected participants based on their self-report of diagnoses, as we felt that it was reasonable to assume that individuals who identified themselves as having a disease did carry that diagnosis and therefore would be in a position to discuss their self-care process. Moreover, recruiting participants through administrative data would have been less reliable, since we could not assume that individuals would be aware of all the diagnoses on their medical records. Self-report of chronic disease diagnoses has been shown to agree with medical record diagnoses from $73 \%$ to $83 \%$ of the time. ${ }^{15}$

Patients with any active terminal illness, HIV infection, mental incompetence, or uncontrolled psychiatric illness were excluded. These exclusions were based on the premise that the presence of any of these conditions would dominate the subject's perception of dayto-day self-management of his or her chronic conditions. Since depression also has the potential to alter perception of disease self-management and is common among persons with chronic disease, presence or absence of depression and associated treatment was noted and incorporated into the descriptive results. In addition, since severity of illness may affect perceptions of barriers to self-care, perceived health status was incorporated into the descriptive results as well. The institutional review boards of the University of Colorado Health Sciences Center and participating sites approved the project protocol.

\section{Data collection}

The data were collected using the semistructured oneon-one interview technique of free listing. Free listing is a technique in which a respondent is asked a question about a domain of interest and invited to respond with a 'list' of answers that represent elements in that domain. These answers are tabulated and analyzed for frequency, content, and range of description of the domain of 
interest. Free listing has been recommended as an appropriate method for research involving the initial definition of new domains (in this case barriers to selfcare for persons with more than 1 chronic disease) prior to the development and use of other data collection techniques (such as questionnaires). ${ }^{16}$ It is particularly useful for assuring that the information gathered is relevant for the participants themselves rather than based on presumptions made by the researchers. ${ }^{16}$ Free listing has been used in medical research to assess subjects' knowledge of signs and symptoms of certain illnesses. ${ }^{17,18}$ Free listing provides the additional benefits of allowing immediate subject verification of the content of the data and allowing a 'semiquantified' approach to looking for patterns of responses. During free listing, interviews and recruitment continue until patterns emerge across the lists of responses. This usually occurs at approximately 20 informants. ${ }^{16}$ We chose an individual-interview method based on the assumption that persons with different constellations of illnesses might perceive different barriers to self-care and be more likely to discuss them in a one-on-one setting.

In the interviews, subjects were asked to "List everything you can think of that affects your ability to manage your medical conditions." If necessary, prompts were used, such as: "What makes it easier/more difficult to care for your medical conditions?" and "What would you like to change about what you need to do for your medical conditions?" Both the responses and the order of responses were recorded on index cards and then reviewed with the subject to verify the content of the response. Following the free listing process, we collected descriptive information about demographic characteristics, perceived health status, and number and types of diagnoses. Preliminary analysis of our data revealed a consistent pattern of responses after 11 or 12 interviews. We continued enrolling subjects through 16 interviews and confirmed that these patterns of responses continued and did not expand. ${ }^{16}$

\section{Analysis}

Interviews were conducted by EB (a family physician) and analyzed by DM and DF (a social psychologist and a research associate, respectively, both with expertise in qualitative methodology) and EB. In the first phase of the analysis, we tabulated and sorted subjects' responses, both independently and together, by repeatedly reviewing the items listed during the free listing process and sorting them into groups with similar content. When we disagreed about categorization, we resolved the disagreement by discussion, taking into account the context of the individual interview. If a listed item did not fit into any one category, it was discarded from the analysis. This occurred with 4 out of 211 total respons-

\section{Figure 1. Schematic Representation of the Analytic Methodology}

Interview respondents.

Free listing $\mid \uparrow$ Verification of responses

Collect items listed by respondents.

Sorting of items

collected

QCA to assess

Identify potential barriers to self-care. influence

Review of results $\mid \uparrow$

of health status

Interpret findings in light of components

of the self-management process. ${ }^{8}$

QCA: Quality Comparative Analysis

es. These groups of similar items from the free listing process represent elements in the domain of potential barriers to self-care for this group of respondents. In order to avoid overemphasizing any single element because of the input from just 1 or 2 subjects, the responses were quantified by the number of subjects whose items fell into 1 element rather than by the total number of items in that element.

Since reported health status may affect respondents' perceived barriers to self-care, we analyzed all of the barriers to self-care with regard to the health status of the respondent using Qualitative Comparative Analysis (QCA). Qualitative Comparative Analysis is a technique designed to sort through categorical qualitative data in which multiple factors may be associated with any one outcome and eliminate factors that appear irrelevant to specific outcomes. This process can clarify patterns of factors that may be associated with certain outcomes. ${ }^{19}$ We used QCA to analyze all of the barriers to self-care with regard to "good" or "very good" versus "fair" or "poor" health status. Our goal in this subanalysis was to detect any influence of perceived health status on the elements defined by the respondents.

Finally, we interpreted the potential barriers to selfcare that emerged from our analysis in light of the 4 components of chronic disease self-management to insure that they were, in fact, potential impediments to the self-care process. Figure 1 gives a schematic representation of our analytic methodology.

\section{RESULTS}

Eighteen people responded to the invitation for interviews. Sixteen met enrollment criteria and were subsequently enrolled in the study. Thirteen of the subjects 


\section{Table 1. Demographic Characteristics of Participants ( $n=16$ )}

\begin{tabular}{lc}
\hline Characteristic & Number of participants \\
Female & 13 \\
Male & 3 \\
White & 16 \\
Age & \\
$31-40$ & 1 \\
$41-50$ & 3 \\
$51-60$ & 3 \\
$61-70$ & 4 \\
Older than 70 & 5 \\
Education & \\
$\quad$ High school graduate & 2 \\
Some college & 7 \\
College graduate & 5 \\
Post-college & 2 \\
Annual income & \\
$<\$ 15,000$ & 7 \\
$\$ 15,001-\$ 30,000$ & 3 \\
$\$ 30,001-\$ 45,000$ & 2 \\
$\$ 45,001-\$ 60,000$ & 3 \\
No answer & 1 \\
Health insurance & \\
None & 4 \\
Medicare & 1 \\
Medicaid & 2 \\
Managed care & \\
No answer & \\
\hline
\end{tabular}

were female. Subjects' ages ranged from 31 to 40 years to more than 70 years, all had at least a high school education, a majority had low annual household incomes, most were currently not employed, 4 had no health insurance, and all were white. Descriptive demographic information is listed in Table 1.

The participants reported a variety of diagnoses and combinations of comorbidities. The number of chronic diagnoses per person ranged from 2 to 7 with a mean of 4.3 and a median of 4.0. The combinations of comorbidities were heterogeneous. The most common comorbid conditions were osteoarthritis and hypertension (6) and osteoarthritis and respiratory disease (6). However, in all cases these combinations occurred in combination with a variety of other conditions. Eight of the subjects reported their health status as "good" or "very good," and 8 as "fair" or "poor." None reported "excellent" health status. Fourteen out of 16 reported having at some point been diagnosed with depression, and 6 of those reported current treatment for depression. Table 2 lists the disease characteristics of the subject population.

Sorting the responses to the free listing process revealed 15 potential barriers to self-care in this group of respondents. In some cases the barrier to self-care
Table 2. Disease Characteristics of Participants $(n=16)^{*}$

\begin{tabular}{lc}
\hline Characteristic & Number of participants \\
Medical condition $^{\dagger}$ & \\
Osteoarthritis & 12 \\
Hypertension & 11 \\
Chronic bronchitis, emphysema, asthma & 7 \\
Diabetes & 5 \\
Other musculoskeletal diagnosis & 4 \\
Other psychiatric diagnosis & 4 \\
Vision problem & 4 \\
Coronary artery disease & 3 \\
Migraine & 3 \\
Obesity & 2 \\
Gastroesophageal reflux & 2 \\
Congestive heart failure & 1 \\
Depression, ever & 14 \\
Current treatment & 6 \\
No current treatment & 8 \\
Overall health status & \\
Excellent & 0 \\
Very good & 1 \\
Good & 7 \\
Fair & 7 \\
Poor & 1 \\
* Mean number of conditions, 4.3 (range, 3-7). Median number of conditions, 4.0. \\
† Each of the following diagnoses was reported by one subject: arrhythmia, \\
stroke, chronic sinusitis, varicose veins, neuropathy, alcoholism, incontinence. \\
\hline
\end{tabular}

was illustrated by mention of a coping mechanism used to overcome the potential barrier. These are listed in Table 3 along with representative quotations. Several of these potential barriers to self-care are of particular interest because of the noted influence of comorbidity:

Fourteen out of the 16 respondents reported that symptoms of one of their conditions or lifestyle changes necessitated by one of their conditions interfered with self-care for another condition. For example, "... can't exercise for diabetes due to the breathing .." or "... when asthma is bad ... makes arthritis harder to manage [as shortness of breath further impairs mobility]." Whereas lifestyle changes for 2 conditions often seemed incompatible: "... changing diet to low fat makes constipation worse ..." Several respondents mentioned that [psychological] "stress" from one condition would often aggravate another.

Nine subjects mentioned compound effects of medications as interfering with care: in these cases either therapeutic effects or adverse effects of a medication for one condition aggravated symptoms of another. For example: "... cough medicine makes blood pressure worse ..." or "... taking medicine for one messes up another condition. ..." Other medication concerns included discouragement about the total burden of 
medications and the complexity of the medication schedule.

Eight subjects cited a single dominant condition as an impediment to the care of other conditions. One participant explained that "... loss of vision is worse than COPD or arthritis," whereas another mentioned "I'd rather the arthritis than the asthma." Dominant diseases listed as interfering with self-management of comorbid conditions included vision troubles, diabetes, depression and asthma. There was no pattern to these dominant conditions. For example, chronic obstructive pulmonary disease was listed as the "... most troublesome ..." and "... the easiest to ignore. ..."

Qualitative Comparative Analysis revealed that all potential barriers to self-care were mentioned in some combination by subjects with both better and worse perceived health status. In other words, for this study population, these elements of the domain of barriers to self-care do not appear to be disproportionately influenced by either better or worse perceived health status.

Finally, all of these elements have the potential to interfere with the self-management process and therefore may be considered potential barriers to self-care.

\section{DISCUSSION}

The need to care for a growing population of patients with comorbid chronic diseases mandates that we develop new, multidimensional models that incorporate the patient's perspective in managing his or her care. Recent articles on chronic care emphasize the importance of self-care: Wagner et al identified selfcare as a major component of chronic disease management and specifically mentioned the need to identify barriers to reaching self-care goals, and Glasgow described identifying barriers as part of successful selfmanagement programs. ${ }^{20,21}$ Since we cannot successfully design self-care interventions for persons with comorbid chronic diseases until we assess their needs, we chose to investigate barriers to self-care in persons with more than 1 chronic disease. Our study population reported a range of barriers to self-care and associated adaptations to these barriers that reflect both the nature of chronic disease and the management of multiple morbidities. Furthermore, these barriers to self-care are relevant for persons across a range of perceived health strata and may interact with various components of the self-management process.

In previous qualitative studies on barriers to self-care that have focused on single diseases, respondents reported multiple barriers to self-care, including concerns about knowledge deficits, physical and financial access to care, adverse effects of medications, negative emotions, personal struggles and difficulties with lifestyle changes. ${ }^{9-13}$ Since these barriers to self-care have been reported in multiple scenarios, they do not appear to be specific to the conditions studied. However, their relative importance may vary for different diseases and comorbidities.

Where comorbidity was mentioned in previous studies, it was also seen as a barrier to self-care, but not investigated in detail. In one study on pain management in the elderly, the presence of additional medical conditions was specifically mentioned as a barrier in the self-management of chronic pain $^{11}$; and an investigation into 
barriers to self-care in persons with congestive heart failure reported comorbidities as interfering with selfmanagement of heart failure. ${ }^{13}$

Our study question allowed respondents to elaborate on issues that result from attempting to self-manage coexisting medical conditions. In addition to mentioning barriers to self-care previously reported by persons with single chronic conditions, they reported that compound effects of conditions, multiple medication issues and the burden of caring for a single dominant condition commonly interfered with effective self-care. Compound effects of conditions often centered on physical limitations or complex recommendations for lifestyle changes. This barrier to self-care may interfere with the self-management task of engaging in activities that promote physical and psychological health. ${ }^{8}$ In these reports, patients who had the confidence to tackle disease-specific recommendations were often stymied by the competing demands of seemingly incompatible self-management tasks. Although only a few respondents implied a direct connection between medication concerns and their medication adherence, significant declines in adherence have been associated with increases in complexity or number of medications. ${ }^{22,23}$ Some participants reported an overwhelming effect of one single disease as specifically interfering with the self-care of comorbid conditions. This situation could certainly have a negative effect on participants' ability to manage the impact of illness on physical, psychological and social functioning. ${ }^{8}$

The fact that respondents mentioned, on average, more than 7 barriers to self-care highlights the synergistic nature of coping with chronic disease self-management. Just as providers report competing demands in caring for patients with multiple medical illnesses, ${ }^{24,25}$ patients attempting to manage multiple conditions perceive their own competing demands from selfcare for their comorbidities. "Dual task theory," which proposes that individuals perform tasks first in which they have the greatest emotional investment, has been offered as an explanation for physicians' behavior in triaging acute symptoms and illness at the expense of chronic care ${ }^{7}$; our study suggests that this theory could easily be applied to patients as well. Individuals may perform self-care tasks for conditions in which they have an emotional investment at the expense of others. If providers are unaware of the burden of suggested treatments, the barriers patients face in implementing them, and the priorities patients place on management of their health conditions, their overall health may either fail to improve or even decline as a result of therapeutic efforts.

Our investigation was limited by the recruitment process. In our efforts to enroll subjects who were aware of their medical diagnoses, we relied on recruitment through passive advertising in primary care offices. This undoubtedly attracted a group of participants who were aware of some of the management issues surrounding their diagnoses and were willing to discuss them. In addition, our study population is demographically limited — specifically by racial/ethnic group and somewhat by gender. Barriers to self-care for persons who have different demographic characteristics, are less knowledgeable about their medical conditions, are cared for primarily by specialists, and/or are not receiving regular medical care may differ. Finally, we did not assess marital status and other aspects of the respondents' living situations. Level of social support will affect perceived barriers to self-care as well. Incorporating the information we have gathered into a more quantitative investigation with a larger, more diverse study population would address these concerns.

A majority of our respondents (14/16) reported either a past or current diagnosis of depression, and 6 were currently under treatment. This lifetime prevalence is much higher than the estimated $42 \%$ lifetime history of psychiatric illness in persons with chronic medical illness. ${ }^{26}$ However depressive symptoms may increase with increasing numbers of comorbidities. ${ }^{27}$ The prevalence of depression in our study population may also reflect the recruitment process in that patients with depression may be more likely to identify themselves as having multiple medical conditions or feel inclined to discuss the associated burdens of self-care. As increased severity of depression has been associated with decreases in certain aspects of self-care and with increased severity of chronic illness, ${ }^{28,29}$ the high prevalence of depression in this group may have resulted in increased reports of perceived barriers to self-care.

This initial definition of the domain of barriers to self-care for persons with comorbidities is a first step in exploring the interactive process of self-care of multiple medical conditions. If further quantitative investigations confirm our suggestion that some barriers to self-care are specifically relevant to persons with comorbid chronic disease, the implications are significant. Disease management strategies that are currently based on a single disease models ${ }^{29,30}$ or chronic disease in general ${ }^{3}$ might be altered to incorporate assessments of comorbidity and associated barriers to self-care. Providers' and patients' perceptions of 'competing demands' attributable to multiple diagnoses might be alleviated by a reorganized model that emphasizes collaborative management of physical and emotional well being. Patient and provider recognition of self-care barriers and prioritization of self-care tasks might give patients tools to address specific issues of symptom and medication management. Any interventions that 
ultimately improve the health of persons with comorbid chronic illness have the potential to reduce individual disability, improve individual quality of life, and affect the organization of care.

To read commentaries or to post a response to this article, see the online version at http://www.annfammed.org/cgi/full/1/1/15.

Key words: Comorbidity, Self-care, Chronic Disease, PBRN

Received October 15, 2002; accepted January 28, 2003.

This project was completed while Dr. Bayliss was primary care research fellow at the University of Colorado Health Sciences Center. Portions of this manuscript were presented in poster format at the NRSA2002 Trainees Research Conference; June 22, 2002, Washington, DC , and in paper format at the North American Primary Care Research Group Meeting, November, 17-20, 2002, New Orleans, La.

\section{Financial Support}

This project was supported by NRSA training grant HP-10006-09 5 T 32, Health Resources Services Administration, and by Research Stimulation Grant G0204RS, American Academy of Family Physicians Foundation.

\section{Acknowledgements}

We would like to thank the CaReNet primary care research network for the opportunity to conduct this study in four of their practices, and Russell E. Glasgow $\mathrm{PhD}$ for his review of the manuscript and helpful comments.

\section{REFERENCES}

1. Anderson GF. Partnership for solutions. Better lives for people with chronic conditions. 2000. Robert Wood Johnson Foundation and Johns Hopkins University. Available at: http:// www.partnershipfor solutions.org/. Accessed March 2003.

2. Hoffman C, Rice D, Sung HY. Persons with chronic conditions. Their prevalence and costs. JAMA 1996;276:1473-1479.

3. Lorig KR Sobel DS, Stewart AL, et al. Evidence suggesting that a chronic disease self-management program can improve health status while reducing hospitalization: a randomized trial. Med Care 1999;37:5-14.

4. Patrick DL, Dinne S, Engelberg RA, Pearlman RA. Functional status and perceived quality of life in adults with and without chronic conditions. J Clin Epidemiol 2000;53:779-785.

5. Stewart AL, GreenfieldS, Hays RD, et al. Functional status and wellbeing of patients with chronic conditions. Results from the Medical Outcomes Study. JAMA 1989;262:907-913.

6. Gijsen R, Hoeymans N, Schellevis FG, Ruwaard D, Satariano WA, van den Bos GA. Causes and consequences of comorbidity: a review. J Clin Epidemiol 2001;54:661-674.

7. Wagner EH, Austin BT, Von Korff M. Organizing care for patients with chronic illness. Milbank Q 1996;74:511-544.

8. Clark NM, Becker MH, Janz NK, Lorig K, Rakowski W, Anderson L. Self-management of chronic disease by older adults. A review and questions for research. J Aging Health 1991;3:3-27.
9. Simmons D, Weblemoe T, Voyle J, Prichard A, Leakehe L, Gatland B. Personal barriers to diabetes care: lessons from a multi-ethnic community in New Zealand. Diabet Med 1998;15:958-964.

10. Wdowik MJ, Kendall PA, Haarris MA. College students with diabetes: using focus groups and interviews to determine psychosocial issues and barriers to control. Diabetes Educ 1997;23:558-562.

11. Lansbury G. Chronic pain management: a qualitative study of elderly people's preferred coping strategies and barriers to management. Disabil Rehabil 2000;22:2-14.

12. Buetow S, Goodyear-Smith F, Coster G. Coping strategies in the selfmanagement of chronic heart failure. Fam Pract 2001;18:117-122.

13. Riegel B, Carlson B. Facilitators and barriers to heart failure self-care. Patient Educ Couns 2002;46:287-295.

14. Parnes B, Main DS, Holcomb S, Pace W. Tobacco cessation counseling among underserved patients: a report from CaReNet. J Fam Pract 2002;51:65-69.

15. Martin LM, Leff M, Calonge N, Garrett C, Nelson DE. Validation of self-reported chronic conditions and health services in a managed care population. Am J Prev Med 2000;18:215-218.

16. Weller SC, Romney AK. Systematic Data Collection. Newbury Park, Calif: Sage Publications, 1988.

17. Mehanna S, Winch PJ, Rizkalla NH, el-Sayed HF, Abaza SM. Factors affecting knowledge of the symptoms of schistosomiasis in two rural areas near Ismailia, Egypt. Trop Med Int Health 1997;2(11 suppl):A36-A47.

18. Ryan GW, Martinez H, Pelto GH. Methodological issues for eliciting local signs/symptoms/illness terms associated with acute respiratory illnesses. Arch Med Res 1996;27:359-365.

19. Ragin CC. Using qualitative comparative analysis to study causal complexity. Health Serv Res 1999;34(5 part II):1225-1239.

20. Wagner EH, Austin BT, Davis C, Hindmarsh M, Schaefer J, Bonomi A. Improving chronic illness care: translating evidence into action. Health Aff [Millwood] 2001;20:64-78.

21. Glasgow RE, Orleans $C T$, Wagner EH. Does the chronic care model serve also as a template for improving prevention? Milbank $Q$ 2001;79:579-612.

22. Brown BG, Bardsley J, Poulin D, et al. Moderate dose, three-drug therapy with niacin, lovastatin, and colestipol to reduce low-density lipoprotein cholesterol $<100 \mathrm{mg} / \mathrm{dl}$ in patients with hyperlipidemia and coronary artery disease. Am J Cardiol 1997;80:111-115.

23. Baird MG, Bentley-Taylor MM, Carruthers SG, et al. A study of efficacy, tolerance and compliance of once-daily versus twice-daily metoprolol (Betaloc) in hypertension. Betaloc Compliance Canadian Cooperative Study Group. Clin Invest Med 1984;7:95-102.

24. Nutting PA, Rost K, Smith J, Werner JJ, Elliot C. Competing demands from physical problems: effect on initiating and completing depression care over 6 months. Arch Fam Med 2000;9:1059-1064.

25. Rost K, Nutting P, Smith J, Coyne JC, Cooper-Patrick L, Rubenstein L. The role of competing demands in the treatment provided primary care patients with major depression. Arch Fam Med 2000;9:150-154.

26. Wells KB, Golding JM, Burnaam MA. Psychiatric disorder in a sample of the general population with and without chronic medical conditions. Am J Psychiatry 1988;145:976-981.

27. Lee Y, Choi K, Lee YK. Association of comorbidity with depressive symptoms in community-dwelling older persons. Gerontology 2001;47:254-262

28. Ciechanowski PS, Kaaton WJ, Russo JE. Depression and diabetes: impact of depressive symptoms on adherence, function, and costs. Arch Intern Med 2000;160:3278-3285.

29. Coulehan JL, Schulberg HC, Block MR, Janosky JE, Arenaa VC. Medical comorbidity of major depressive disorder in a primary medical practice. Arch Intern Med 1990;150:2363-2367.

30. Mensing C, Boucher J, Cypress M, et al. National standards for diabetes self-management education. Task Force to Review and Revise the National Standards for Diabetes Self-Management Education Programs. Diabetes Care 2000;23:682-689. 